\title{
A Digital Signal Processing Index of Hand Tremor
}

\author{
Richard Reilly, Student Member, IEEE, Annraoi de Paor \\ Department of Electronic and Electrical Engineering, University College Dublin, \\ Belfield, Dublin 4, Ireland.
}

\begin{abstract}
An index of hand tremor is formulated from the data obtained from the analysis of the cylindrical grip strength. The tremor signal is extracted digitally from the data and the mean square power calculated. The frequency corresponding to half of the mean square power is compared against arthritic sufferers and normals. The application of such an index to the diagnosis and classification of Vibration White Finger is discussed.
\end{abstract}

\section{INTRODUCTION}

According to the Arthritis Foundation of Ireland [1] arthritic diseases affect over 500,000 people in Ireland and between 150,000 and 200,000 seriously. This represents 15 $20 \%$ of the country's population. It has been reported that over 40 million Americans suffer from Osteoarthritis [2]. The loss of movement is a major occupational problem in the United States costing on average 10 billion dollars a year [3]. Neumann et al [4] believe $3-6 \%$ of the population of the developed countries suffer from severe arthritis.

The symptoms of rheumatoid arthritis can often, but not always, be controlled by the use of steroids and other antiinflammatory drugs. However they will not reverse the medical situation. Current treatment centres on the reduction of pain, minimisation of joint destruction and the improvement of function. The three main areas of treatment include: medication, physical therapy and surgery.

Prostheses are available for replacement of finger and metacarpal joints. Arthrodesis (surgical immobilisation of a joint so that the bones grow solidly together) of the thumb interphalangeal joint accompanied by prosthetic surgery to the metacarpal joints, is a procedure commonly used to restore a precision grip to severely deformed sufferers. Rheumatoid sufferers are also encouraged, whenever possible, to build up strength while maintaining joint protection. The onset of rheumatoid arthritis can occur at any age, young or old, with middle aged women running a higher risk of acquiring the disease than other groups.

With the availability of new medication, it is vital to track the progress of this disease. The current assessment method for rheumatoid arthritis is achieved by squeezing the affected joints and scoring each with a value of one to three, depending how much the patient flinches. The diagnostic assessment for De Quervain's Tenosynovitis, which is a muscle tendon complaint, involving any pinching motion, centres about Finkelsteins's Test. During this appraisal, the thumb is wrapped with the other four fingers against the palm. The physician or therapist pulls the fist in an ulnar direction. Any pain during this manoeuvre is considered as a positive test for the disease.

A more efficient index of arthritis is required to evaluate the state of health of patients at different stages during treatment. The evaluation of the cylindrical grip and the grips generated between the thumb and each digit are the initial stages in the formation of such an index.

\section{CYLINDRICAL GRIP MONTTORING}

The measurement of the cylindrical grip is made by incorporating two Force Sensing Resistors (FSR) sensors [5] in a parallel arrangement within the jaws of a lightweight mechanical clamp. Within this layout, the FSR is subjected to increasing pressure when a gripping force is applied to the opposite end of the transducer. Two transducers are employed, due to saturation of the dynamic range of a single sensor by a strong grip. The FSRs are pretensioned by the trimming screws located on each corner of the rectangular jaws. The sensitivity is adjusted so that a very weak force can achieve a noticable variation in voltage, without being absorbed by the mechanical structure. The mechanical construction of the transducer is shown in fig. 1. 


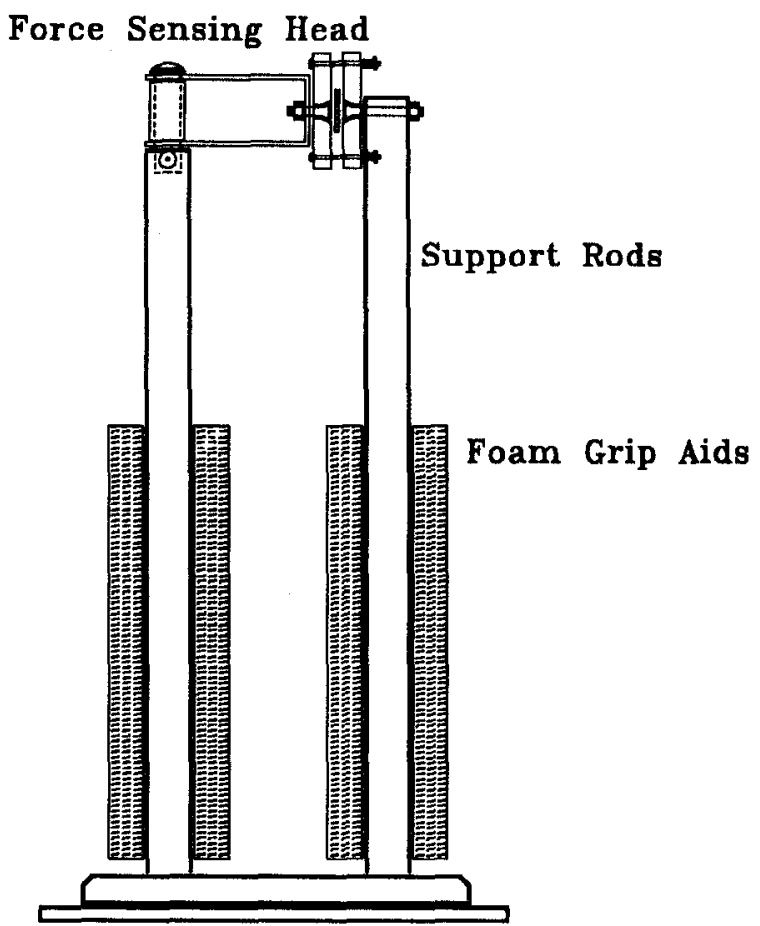

(a)

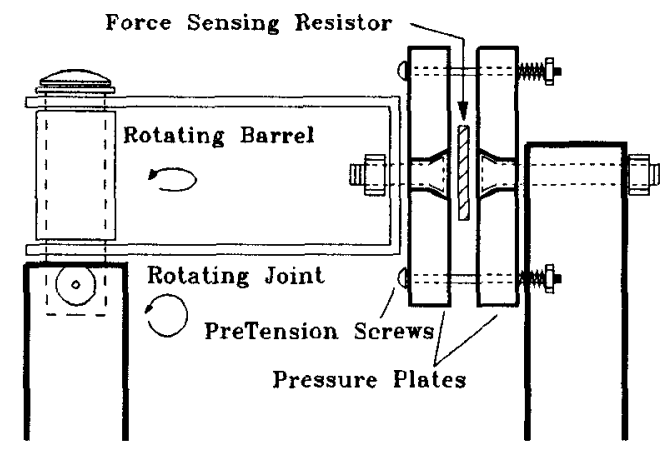

(b)

Fig. 1. (a) Side view of the grip transducer incorporating FSR Sensors. (b) detail of the pressure plate head of the transducer.

An interface was designed to link the amplifier circuit, of which the FSR is an integral part, and an IBM PC. The variation in voltage due to the exertion of force, is digitised into 12-bits, the ADC being synchronised to the controlling PC. Preceding the ADC is a 8 channel analog multiplexer allowing the monitoring of hand function while conducting routine and complex manoeuvres. Eight independent channels exist, allowing eight sensors to be placed at anatomical sites on the hand, or alternatively at sites on an object to be grasped. One channel is dedicated to grip measurement, leaving seven available for hand function testing. A typical grip response for an arthritic sufferer is shown in Fig. 2.

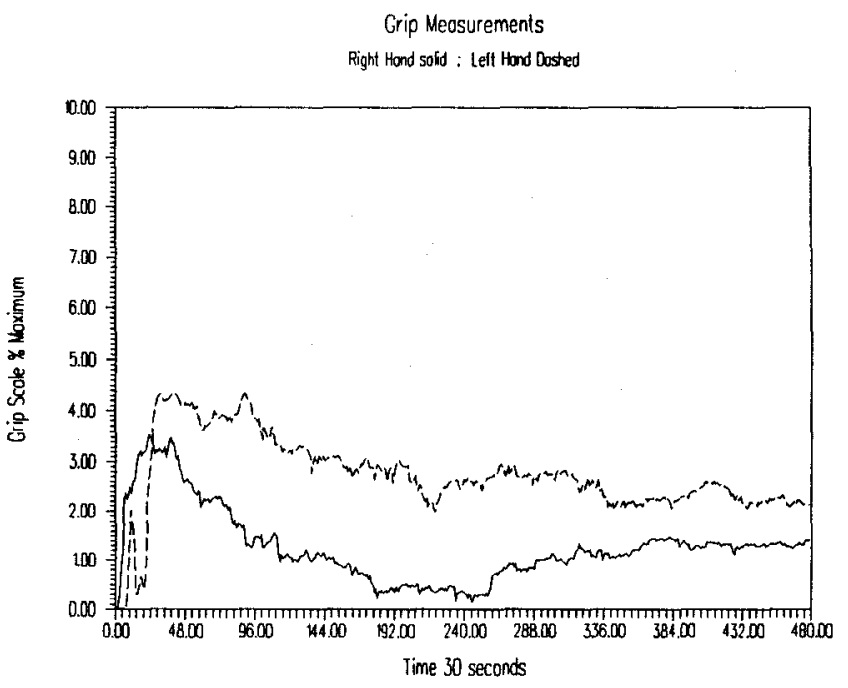

FIG. 2. Typical grip strength variation with time of an Arthritic Sufferer.

\section{EXTRACTION OF MEAN SOUARE POWER}

An analysis scheme has been developed for the extraction of an index of hand tremor. The raw grip data $r(t)$ is passed through a digital lowpass filter with a cut-off frequency of $0.1 \mathrm{~Hz}$, generating $r^{\prime}(t)$. On extracting the residuals an analysis of the energy components contained in $s(t)$ is carried out.

$$
s(t)=r(t)-r^{\prime}(t)
$$

The series $s(t)$ is normalised. This normalised sequence is passed through a lowpass filter whose cut-off frequency is initially set at the nyquist frequency. The mean square power is calculated for the resulting filtered sequence. The filter is adjusted so as to decrease the cut-off frequency. The half power frequency is taken as an index of hand tremor for comparative purposes.

The grip response was assessed under blind subject conditions. The subjects were asked to grip the transducer with the forearm relaxed in a horizontal position. During the testing period of 40 seconds, the subject was not shown the monitor displaying the data, nor told of his or her performance.

\section{RESULTS}

The half power frequency was calculated for each hand of eight subjects and is displayed in histogram form for the left and right hand in fig 3. 


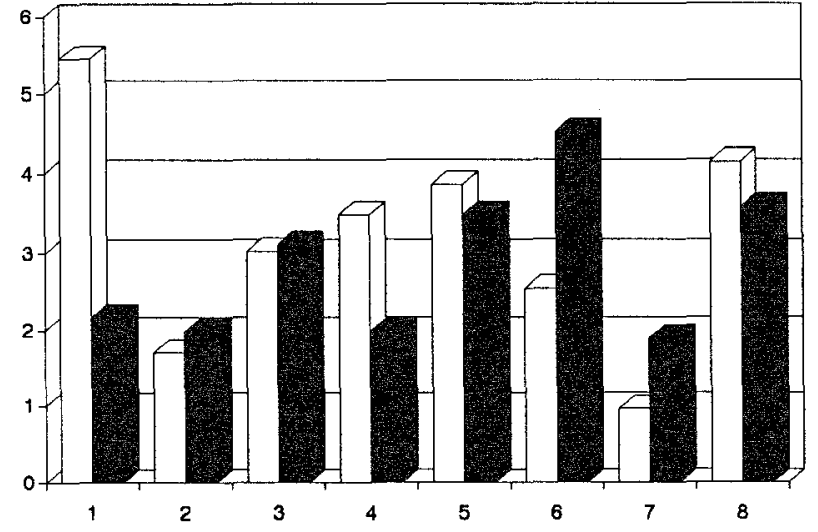

Fig. 3. Half Power Frequency of the Left (white) and Right (black) hand

Of the eight subjects represented here, six are arthritic and two experimental controls. Subject 1 , an arthritic sufferer, is seen to have the highest mean square power frequency value. The maximum grips for both left and right hands of this subject are identical, indicating that muscular and bone structure are sufficient in both hands to achieve a grip, average for arthritic sufferers. The tremor in the left hand is such that it manifests itself at a higher frequency, than that of the right.

Subjects 2 and 3 are experimental controls. Subjects 4, 5 and 8 report higher left hand half mean square power frequencies than right. These subjects responded with stronger grip values for left hand measurements than right hand ones. Subjects 6 and 7 are both right handed. Subject 7 has undergone treatment for rheumatoid arthritis over a period of one year. This has successfully reduced the level of swelling and pain, however Osteoarthritis has now set in at the damaged joints. This allowed an increased grip strength in both hands with reduced tremor, however ankylosing of the MCP joints may be responsible for the insustainability or falling of the grip response with time. Of the eight subjects described here, three are female. The analysis is valid for both sexes, due to grip strength being normalised.

\section{VIBRATION WHITE FINGER}

The use of grip strength and half power index as an assessment method for VWF was carried on 30 machinists. In a study conducted, the index criteria successfully found $60 \%$ of VWF sufferers and pin pointed eight other potential sufferers.
It is imperative for such an index to have the ability to discriminate between vibration workers with and without VWF, rather than between vibration and non-vibration workers. To fully compare those reporting VWF with those who do not, a full history of vibration exposure must be taken into account along with joint damage. The assessment of grip is valid, due to the construction of the transducer. The design is such that the average hand cannot envelope the gripping rods, but can only use the distal and middle phalanges and the proximal phalanx of the thumb for grip. The vasospasm and sensory loss experienced in the distal phalanges of VWF sufferers would reduce their capacity for a sustainable grip over $\mathbf{4 0}$ seconds. Further refinement of the assessment procedure along with a history of exposure to vibration, may prove an alternative to the classical tuning fork or electro-vibration assessment tests [6], [7], [8].

\section{CONClusion}

There is an indication from these results that a difference in half mean square power frequency may be due to rheumatoid arthritis. It is imperative to bear in mind that a history of hand injury or joint damage may be responsible of the differences in half power frequency. The genetic history and the emotional state of the patient are also important in the accurate assessment of arthritis.

\section{REFERENCES}

[1] Arthritis Foundation of Ireland. Understanding Arthritis, Dublin, 1989.

[2] Corman L.C., Bell C.L., Edwards N.L., Harmon C.E. Rheumatology for the House Officer. Williams \& Wilkens, Baltimore, 1990.

[3] American College of Rheumatology. Dictionary of Rheumatic Diseases Volume I : Signs and Symptoms. Associates International, New York, 1985.

[4] Neumann V, Wright V. Rheumatoid Arthritis and Other Inflammatory Arhropathies. From Rehabilitation of the Physically Disabled Adult. Cambridge University Press, 1988.

[5] Interlink Electronics Europe : Product Design Guide, B.P.8, Zone Industrielle, L-6401 Echternach G.D. de Luxembourg.

[6] Alann E.V. Brown G.E. Raynaud's disease : clinical study of 147 cases. Journal of American Medical Association. No.99, 1472., 1932.

[7] Roath S. Raynaud's Disorder(s): Clinical and Pathophysiological Features, from Raynaud's : a Guide for Health Professionals . Edited by S. Roath, Chapman and Hall, London, 1989.

[8] Hayward R., Griffin M. Sensory Aspects of Raynaud's Phenomenon, from Raynaud's : a Guide for Health Professionals. Edited by $\mathbf{S}$. Roath, Chapman and Hall, London, 1989. 